\title{
The value of non-invasive myocardial work indices derived from left ventricular pressure-strain loops in predicting the response to cardiac resynchronization therapy
}

\author{
Mengruo Zhu ${ }^{1}$, Yanan Wang ${ }^{1}$, Yufei Cheng ${ }^{1}$, Yangang Su${ }^{2}$, Haiyan Chen ${ }^{1}$, Xianhong Shu ${ }^{1,2}$ \\ ${ }^{1}$ Department of Echocardiography, Zhongshan Hospital, Fudan University, Shanghai, China; ${ }^{2}$ Department of Cardiology, Zhongshan Hospital, \\ Fudan University, Shanghai, China \\ Correspondence to: Xianhong Shu, MD, PhD. Department of Echocardiography, Zhongshan Hospital, Fudan University, 180 Fenglin Road, Shanghai \\ 200032, China. Email: shu.xianhong@zs-hospital.sh.cn; Haiyan Chen, MD. Department of Echocardiography, Zhongshan Hospital, Fudan \\ University, 180 Fenglin Road, Shanghai 200032, China. Email: chenhaiyan212@126.com.
}

Background: Non-invasive left ventricular (LV) pressure-strain loops (PSLs), which are generated by combining LV longitudinal strain with brachial artery blood pressure, provide a novel method of quantifying global and segmental myocardial work $(M W)$ indices with potential advantages over conventional echocardiographic strain data, which suffers from being load-dependent. This method has been recently introduced in echocardiographic software, enhancing the efficiency of MW calculations. This study aimed to evaluate the role of non-invasive MW indices derived from LV PSLs in predicting cardiac resynchronization therapy (CRT) response.

Methods: A total of 106 heart failure (HF) patients scheduled for CRT were included in the MW analysis. Global and segmental (septal and lateral at the mid-ventricular level) MW indices were assessed before CRT and at a 6-month follow-up. Response to CRT was defined as $\geq 15 \%$ reduction in LV end-systolic volume and $\geq 1$ NYHA functional class improvement at 6-month follow-up compared to baseline.

Results: CRT response was observed in 78 (74\%) patients. At baseline, the global work index (GWI) and global constructive work (GCW) were significantly higher in CRT responders than in non-responders (both $\mathrm{P}<0.05)$. Furthermore, responders exhibited significantly higher mid lateral $\mathrm{MW}$ and mid lateral constructive work $(\mathrm{CW})$ (both $\mathrm{P}<0.001)$, but significantly lower mid septal MWI and mid septal myocardial work efficiency (MWE) than non-responders (all $\mathrm{P}<0.01)$. Baseline mid septal MWE (OR 0.975, 95\% CI: 0.959-0.990, P=0.002) and mid lateral MWI (OR 1.003, 95\% CI: 1.002-1.004, P<0.001) were identified as independent predictors of CRT response in multivariate regression analysis. Mid septal MWE $\leq 42 \%$ combined with mid lateral MWI $\geq 740 \mathrm{mmHg} \%$ predicted CRT response, with an optimal sensitivity of $79 \%$ and specificity of $82 \%$ [area under the receiver operating characteristic curve (AUC) $=0.830, \mathrm{P}<0.001$ ].

Conclusions: Assessment of MW indices before CRT could identify the marked imbalance in LV MW distribution and can be widely used as a reliable complementary tool for guiding patient selection for CRT in clinical practice.

Keywords: Cardiac resynchronization therapy (CRT); myocardial work; non-invasive; pressure-strain loop; segmental heterogeneity

Submitted Jun 13, 2020. Accepted for publication Nov 13, 2020.

doi: 10.21037 /qims-20-754

View this article at: http://dx.doi.org/10.21037/qims-20-754 


\section{Introduction}

Cardiac resynchronization therapy (CRT) is a wellestablished treatment for symptomatic patients with dyssynchronous heart failure (HF) (1); however, nearly $30 \%$ of patients undergoing CRT do not respond favorably to therapy (2). As strain suffers from being load-dependent $(3,4)$, its application is limited in this special HF population characterized by left ventricular (LV) dyssynchrony, as early and late activated segments contract at different times in different loading states. LV dyssynchrony is mainly associated with marked imbalance in segmental myocardial work (MW) distribution. Therefore, analysis of global and segmental MW may provide novel insights into the cardiac mechanics of patients with dyssynchronous HF, and may be useful for guiding patient selection for CRT. The MW derived from invasive $\mathrm{LV}$ pressure-volume/strain loops measured during coronary angiography have been developed for many years. Nevertheless, its invasive nature limits its widespread use in clinical practice.

Recently, a new non-invasive approach to evaluate MW was introduced in echocardiographic software. MW augments speckle tracking echocardiography by taking dynamic LV pressure into account, which further facilitates the interpretation of LV longitudinal strain (LS) traces concerning LV pressure dynamics by providing excellent visualization of pressure-strain loops (PSLs). It also adds an important dimension to the evaluation of $\mathrm{LV}$ function. The inclusion of afterload data in the assessment of myocardial performance has the advantage of limiting the variability induced by loading status (5). Both animal experiments and clinical studies have demonstrated the validity of this method $(6,7)$.

The present study aimed to explore the role of baseline $M W$ indices derived from non-invasive LV PSLs in the prediction of CRT response and to evaluate the changes in MW indices at follow-up after CRT.

\section{Methods}

\section{Study population}

A total of 106 consecutive HF patients who were first scheduled for CRT were retrospectively recruited from August 2018 to May 2019. The indication for CRT based on the 2016 European Society of Cardiology (ESC) guidelines (8) were as follows: symptomatic HF patients in sinus rhythm with a QRS duration $\geq 130 \mathrm{~ms}$ and left ventricular ejection fraction (LVEF) $\leq 35 \%$, and New
York Heart Association (NYHA) functional class II-IV despite optimal medical treatment. Patients were excluded from the study for the following reasons: LV outflow tract obstruction (for example, obstructive hypertrophic cardiomyopathy), aortic stenosis, prosthetic aortic valve replacement, and any other cardiac pathologies that could induce a pressure gradient between the aorta and the LV, atrial fibrillation, narrow QRS, a history of acute myocardial infarction or cardiac surgery, absence of clinical follow-up, and/or poor echocardiographic image quality. MW analysis was feasible in $98 \%$ of patients, and only 2 patients were excluded for incomplete MW analysis due to acquisition with inadequate imaging quality. Left bundle branch block (LBBB) was diagnosed according to the criteria proposed by the 2013 ESC guidelines (9) of the Class 1 Recommendation for CRT, namely, a wide QRS duration with QS or $\mathrm{rS}$ in V1, broad (frequently notched or slurred) $\mathrm{R}$ wave in leads $\mathrm{I}$, aVL, V5, or V6, and absence of q waves in leads V5 and V6.

All HF patients, being on stable and optimal medical treatment according to the ESC guidelines (8), underwent baseline and 6-month follow-up evaluations. Response to CRT was defined as a reduction of left ventricular endsystolic volume (LVESV) $\geq 15 \%$ and $\geq 1$ NYHA functional class improvement at the 6-month follow-up $(10,11)$. All responders at the 6-month follow-up satisfied 2 conditions simultaneously: a reduction of LVESV $\geq 15 \%$ and $\geq 1$ NYHA functional class improvement. Patients were classified as non-responders at follow-up after CRT if they showed no improvement in NYHA functional class, were re-hospitalized, underwent cardiac transplantation, had worsening HF, or died.

\section{Echocardiographic acquisition}

With a synchronous ECG connected, all patients underwent standard transthoracic echocardiography using a Vivid E9 or E95 ultrasound system (GE Healthcare, Horten, Norway) equipped with a $3 \mathrm{~S}$ or M5S transducer. Non-invasive cuff blood pressure values were measured and recorded with brachial artery sphygmomanometry immediately before echocardiography. The following parameters were obtained according to the American Society of Echocardiography guidelines (12,13): (I) left ventricular end-diastolic volume (LVEDV); (II) LVESV; (III) LVEF measured using biplane Simpson's method; (IV) interventricular mechanical delay (IVMD); (V) septal-posterior wall motion delay (SPWMD); and (VI) Yu index. 


\section{Offline MW analysis}

Standard two-dimensional apical views (long-axis, fourchamber, and two-chamber) acquired with at least 60 frames per second were digitized during 5 consecutive cardiac cycles in cine-loop format. Views were imported to the dedicated workstation (EchoPAC Version 203, GE Vingmed Ultrasound) for the offline analysis using a vendor-specific module within the Automated Function Imaging (AFI) software.

Following the identification of the timing points of the aortic and mitral valve opening and closure using pulsedwave Doppler recordings in the LV outflow and inflow tract, speckle tracking analysis was performed using 3 standard apical views (long-axis, four-chamber, and two-chamber). The software presented the results of segmental and global LS. Global longitudinal strain (GLS) was calculated from the weighted average of the peak systolic LS of all LV segments. Mechanical dispersion (MD) was calculated as the standard deviation of the time to peak systolic LS of the 17 LV segments.

After strain analysis was completed, the "Myocardial Work" module in AFI was selected to continue the MW analysis. LV strain and systolic blood pressure data were then synchronized through the alignment of valvular timing events, and the software generated global and segmental PSLs automatically. The loop area served as an index of global and segmental MW, namely, the myocardial work index (MWI).

Work was evaluated from mitral valve closure to the mitral valve opening, or in other words, mechanical systole, including isovolumetric relaxation (IVR). During the LV ejection period, MW performed during segmental shortening represented constructive work $(\mathrm{CW})$ of that segment, whereas work performed by the myocardium during segmental elongation represented energy loss, which was defined as wasted work (WW) of that segment. During IVR, this definition was reversed, such that MW during shortening was considered segmental WW, and work during lengthening was considered segmental CW. LV global work index (GWI), global constructive work (GCW), and global wasted work (GWW) were calculated as the average of all segmental values. LV global work efficiency (GWE) was calculated as the sum of CW in all LV segments, divided by the sum of CW and WW in all LV segments, expressed as a percentage. A bull's eye using the 17 -segment model with the values of the segmental MWI and myocardial work efficiency (MWE) was provided (Figure 1).
Segmental MWI was calculated for each LV segment as the difference between the work performed during the shortening of the segment and the work performed during the segment's elongation. Also, a set of values of segmental MW additional indices were provided and could be exported from the software:

* CW was defined as work performed by a segment during shortening in systole adding negative work during IVR lengthening.

* WW was defined as negative work performed by a segment during lengthening in systole adding work performed during shortening in IVR. Although a negative number by definition, WW was presented as a positive number.

* MWE was defined as the ratio between CW and the sum of $C W$ and WW measured at each segment $[\mathrm{CW} /(\mathrm{CW}+\mathrm{WW})]$, expressed as a percentage.

\section{Inter-and intra-observer variability}

Inter- and intra-observer variability of $M W$ indices was assessed using Bland-Altman plots (Figure 2) with data from 30 randomly selected patients. Data was examined twice by one observer who was blinded to the results of the previous measurements, and by a second observer who was blinded to the values obtained by the first observer, respectively.

\section{Statistical analysis}

Continuous data were presented as mean \pm standard deviation, and dichotomous data were presented as numbers and percentages. Comparisons of continuous variables between responders and non-responders were carried out using the independent-samples t-test, while intragroup comparisons were performed using the pairedsamples t-test. Categorical variables were compared using Chi-square tests or Fisher's exact tests when appropriate. Correlations between two continuous variables were analyzed using Pearson's correlation coefficient test. Binary logistic regression analysis was employed to determine the independent predictors of CRT response. The prediction accuracy assessment was conducted by constructing a receiver operating characteristic (ROC) curve. A twosided $\mathrm{P}$ value $<0.05$ was accepted as indicating statistical significance. All analyses were performed with SPSS version 24.0 (SPSS Inc., IBM, Chicago, IL, USA) and MedCalc version 12.5.0.0 (MedCalc Software, Mariakerke, Belgium). 
A
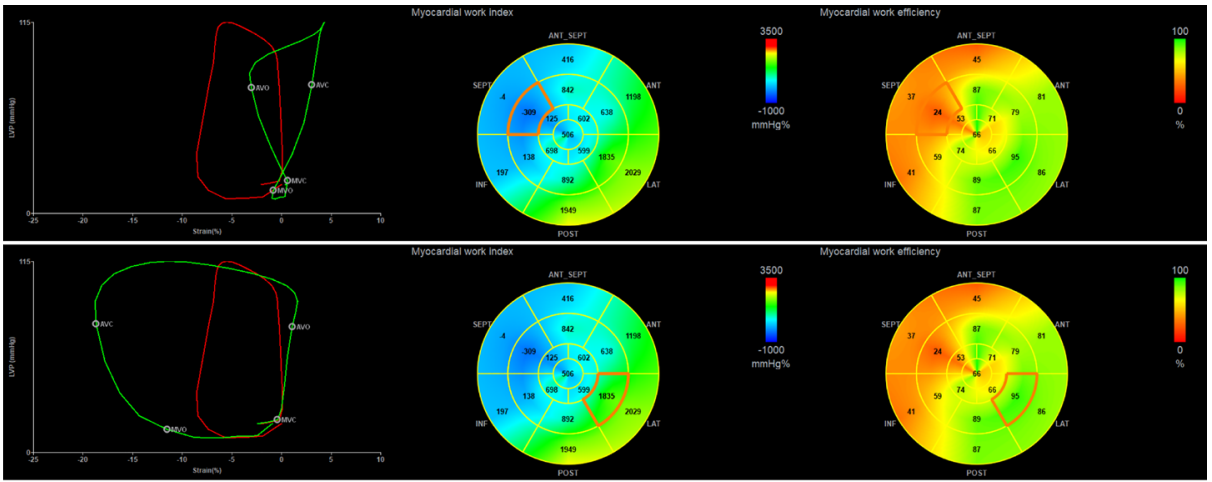

B
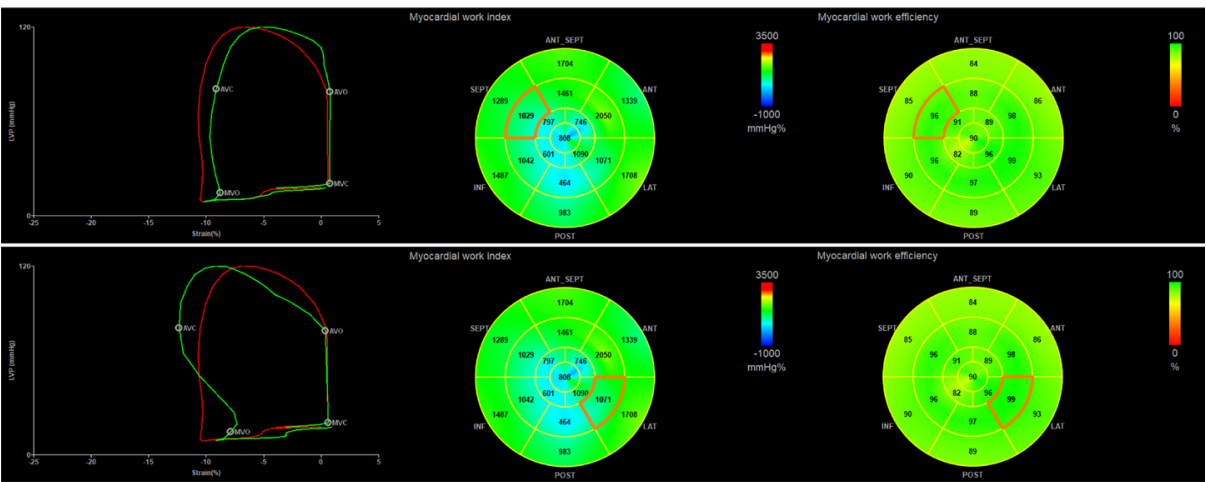

C
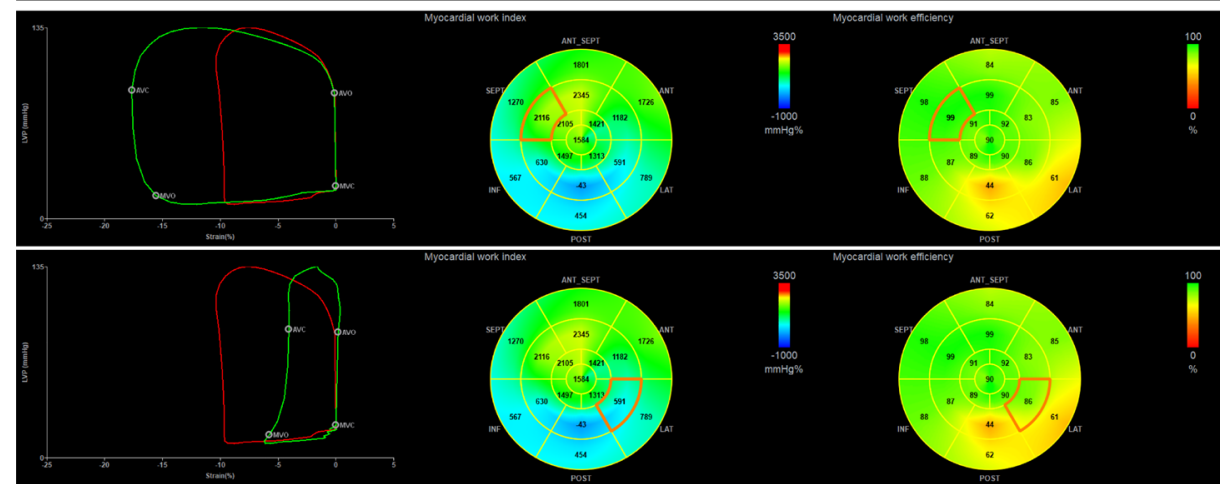

$\mathrm{D}$

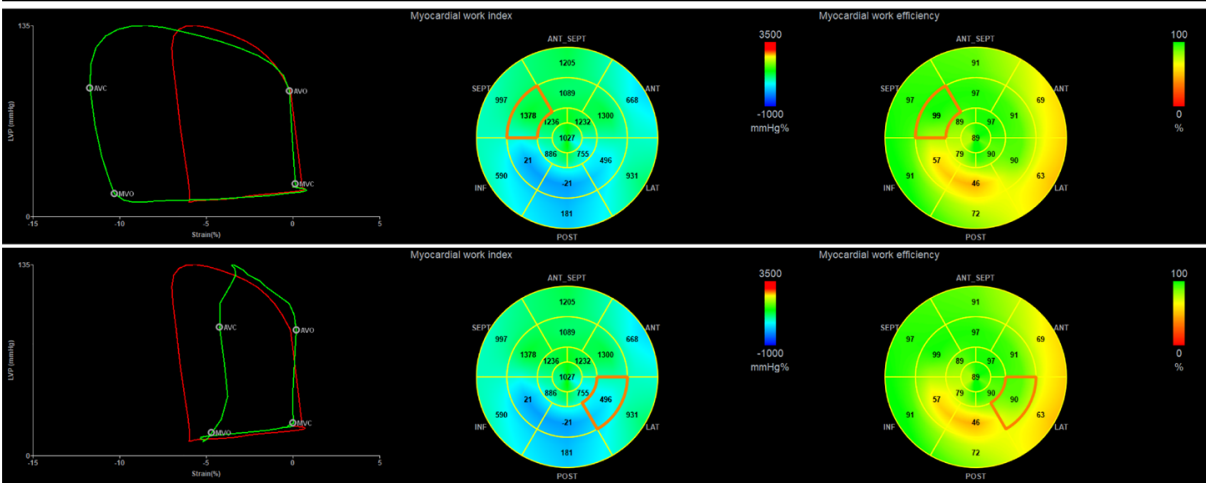

Figure 1 LV PSLs (left), MWI (middle), and MWE (right) of individual examples from CRT responders and non-responders before CRT and at 6-month follow-up after CRT. (A) A CRT responder before CRT; (B) a CRT responder at 6-month follow-up after CRT; (C) a CRT non-responder before CRT; (D) a CRT non-responder at 6-month follow-up after CRT. LV global PSL is represented in red, and segmental (mid septal and mid lateral) PSL is represented in green. LV, left ventricular; PSL, pressure-strain loop; MWE, myocardial work efficiency; MWI, myocardial work index; CRT, cardiac resynchronization therapy. 
A
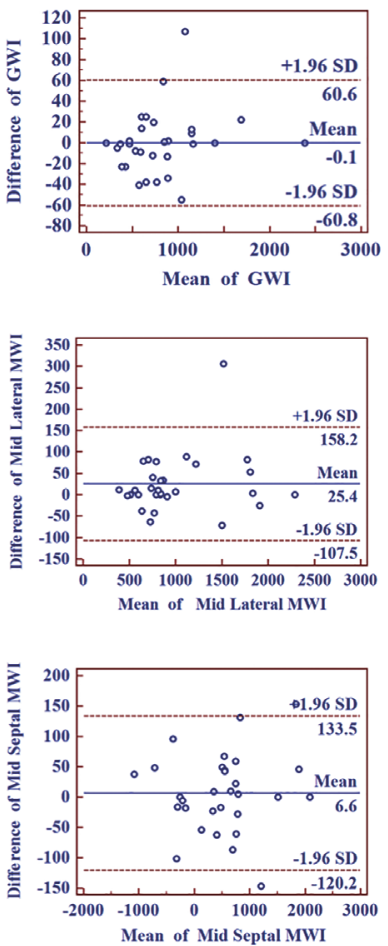

B
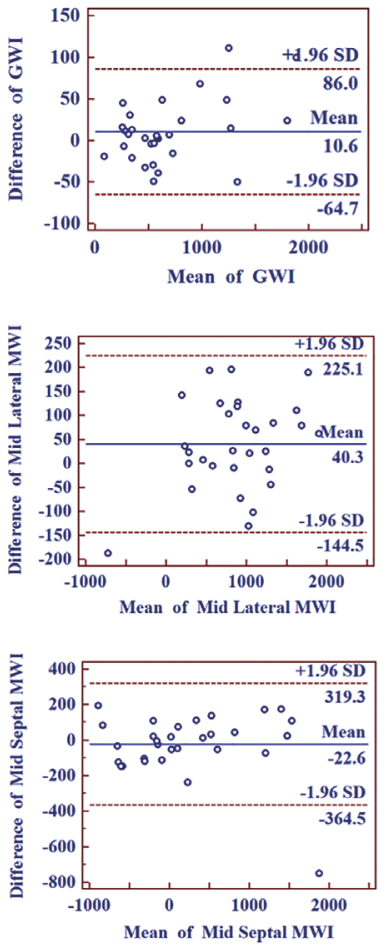
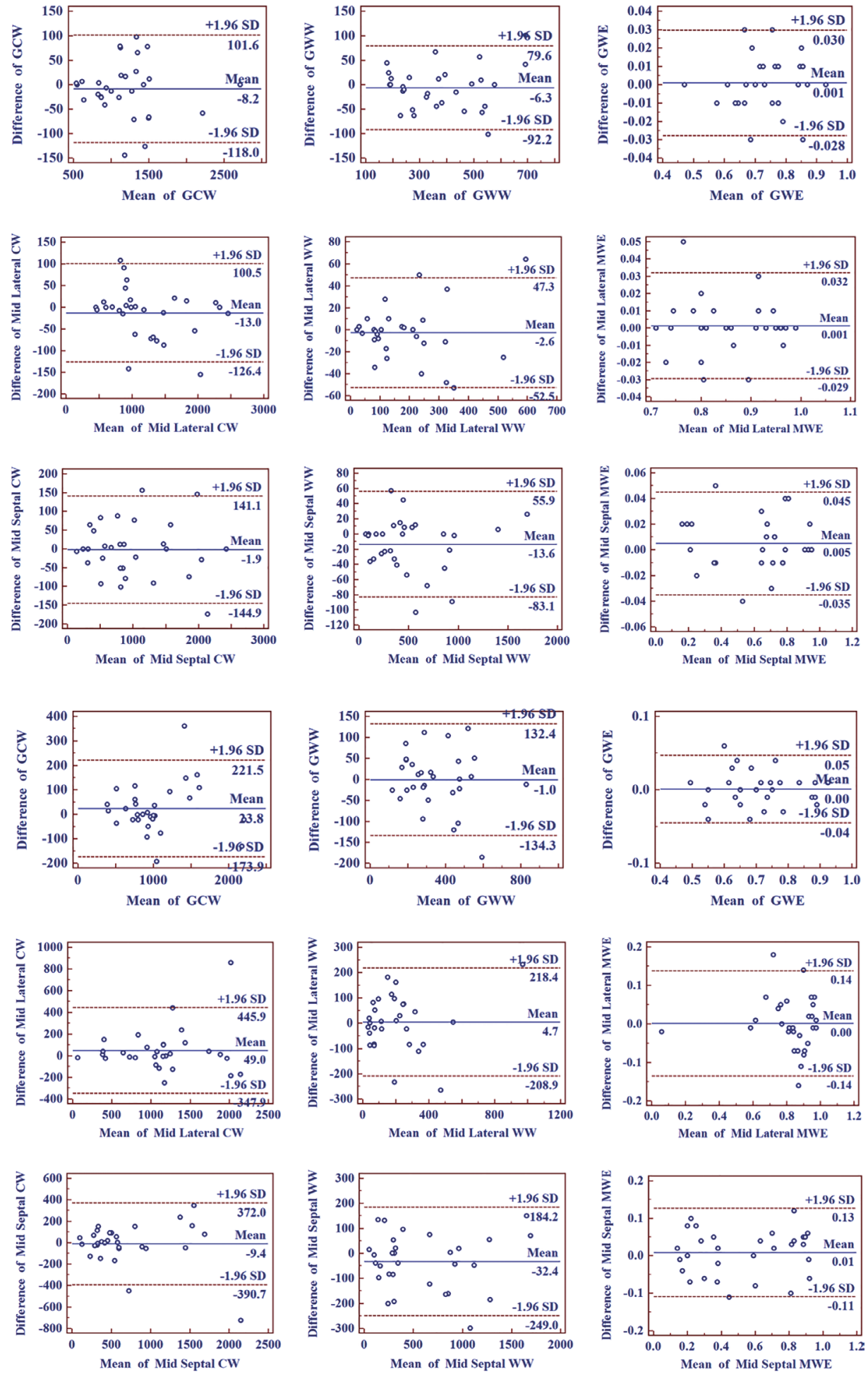

Figure 2 Bland-Altman analysis for intra-observer variability (A) and inter-observer variability (B) of MW indices. Solid line represents bias, and dotted lines represent $95 \%$ limits of agreement for measurements performed in 30 patients. Bias was assessed by the mean of 30 differences of 2 measurements. The $95 \%$ CI: was calculated as \pm 1.96 SD from the mean. CI, confidence interval; CW, constructive work; GCW, global constructive work; GWE, global work efficiency; GWI, global work index; GWW, global wasted work; MW, myocardial work; MWE, myocardial work efficiency; MWI, myocardial work index; SD, standard deviation; WW, wasted work. 
Table 1 Baseline clinical characteristics of CRT responders versus non-responders

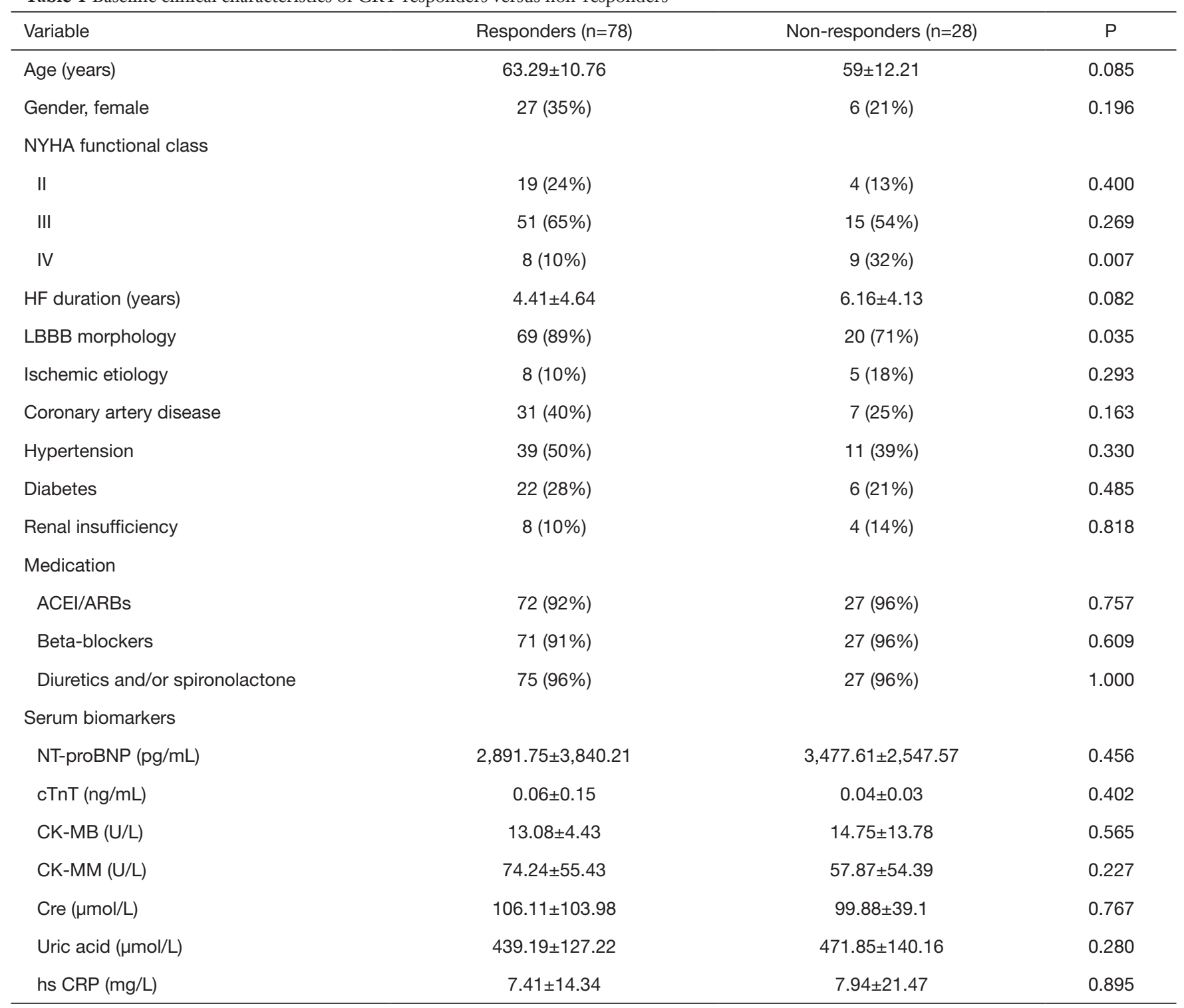

Data are expressed as mean \pm standard deviation or number (\%). ACEI, angiotensin converting enzyme inhibitor; ARB, angiotensin receptor blocker; CK-MB, creatine kinase MB fraction; CK-MM, creatine kinase MM fraction; Cre, creatinine; CRT, cardiac resynchronization therapy; cTnT, cardiac troponin T; HF, heart failure; hs CRP, high-sensitive C-reactive protein; LBBB, Left bundle branch block; NT-proBNP, N-terminal of the prohormone brain natriuretic peptide; NYHA, New York Heart Association.

\section{Results}

\section{Baseline evaluation and changes at follow-up}

At 6-month follow-up, CRT response was found in 78 (74\%) patients, while the other 28 (26\%) patients were classified as non-responders. Baseline clinical characteristics from the overall population are summarized in Table 1 based on CRT response. No significant differences in general clinical characteristics were observed between CRT responders and non-responders, except patients in NYHA functional class IV were more likely to be non-responders $(\mathrm{P}=0.007)$, and LBBB was more prevalent in CRT responders $(\mathrm{P}=0.035)$. Comparisons of other characteristics between CRT responders and non-responders at baseline and at follow-up are shown in Table 2.

At baseline evaluation, CRT responders showed 


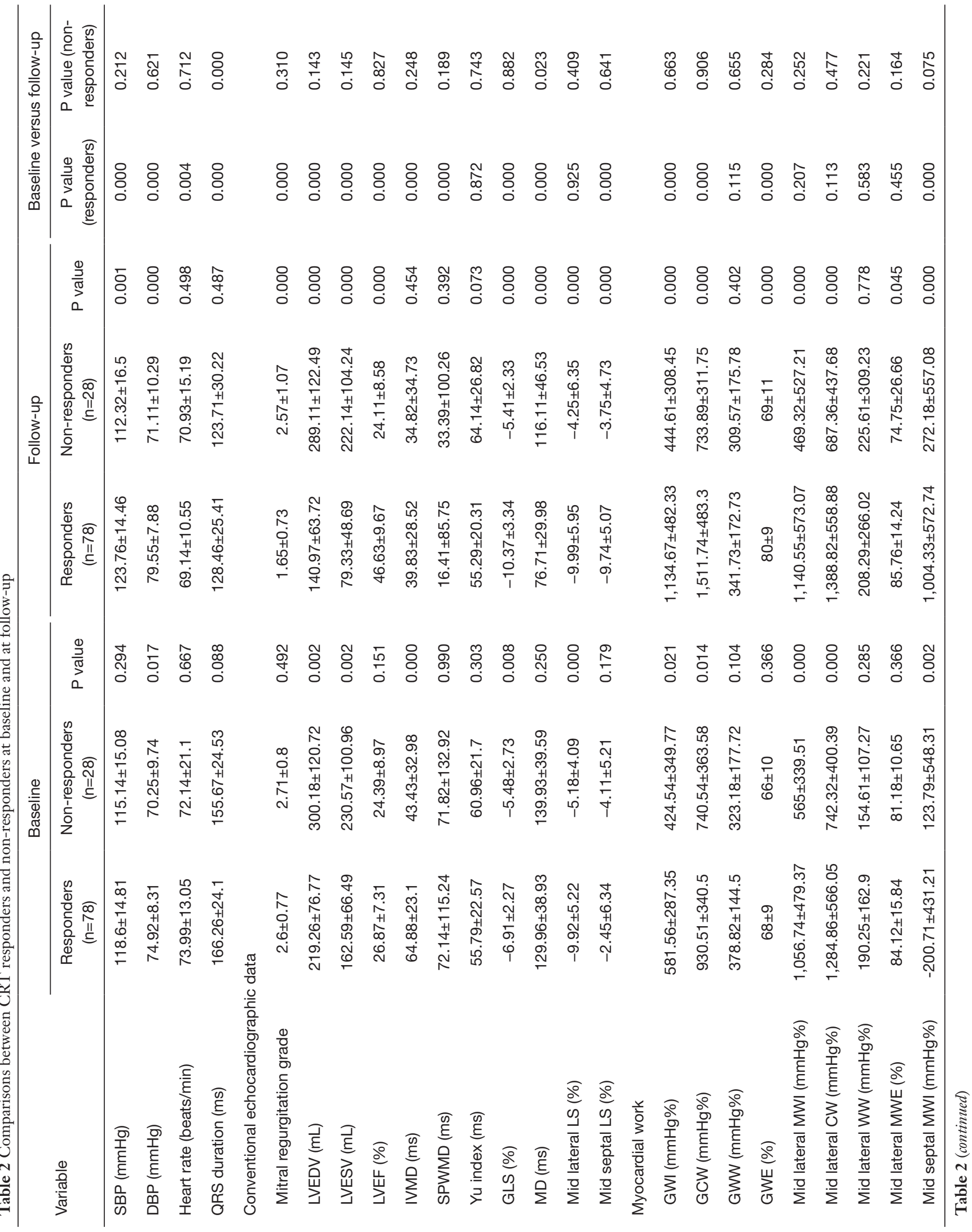




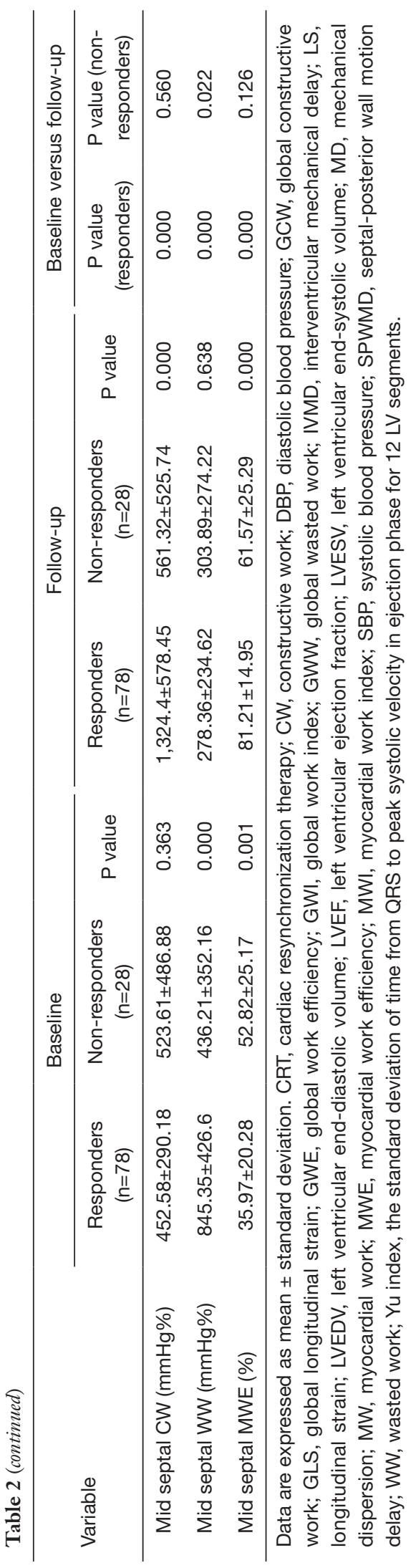

significantly higher diastolic blood pressure $(\mathrm{P}=0.017)$, smaller LVEDV and LVESV (both $\mathrm{P}=0.002$ ), as well as larger IVMD, GLS, and mid lateral LS (all $\mathrm{P}<0.01$ ). Concerning $M W$ indices, GWI and GCW were significantly higher in CRT responders than in nonresponders (both $\mathrm{P}<0.05$ ). Also, CRT responders exhibited significantly higher mid lateral MWI and mid lateral CW (both $\mathrm{P}<0.001$ ), but significantly lower mid septal MWI and mid septal MWE than non-responders (both $\mathrm{P}<0.01$ ). At 6-month follow-up, significant improvements in mid septal MWI, mid septal CW, and mid septal MWE (all $\mathrm{P}<0.001$ ), as well as a significant reduction in mid septal WW $(\mathrm{P}<0.001)$ were observed in CRT responders, accompanied by significant improvements in GWI, GCW, and GWE (all $\mathrm{P}<0.001)$.

\section{Correlations between advanced $M W$ indices and conventional echocardiographic parameters}

The increased values of global MW indices $(\triangle \mathrm{GWI}$, $\triangle \mathrm{GCW}$, and $\triangle \mathrm{GWE})$ and mid septal $\mathrm{MW}$ indices $(\triangle \mathrm{mid}$ septal MWI, $\triangle$ mid septal $C W$, and $\triangle$ mid septal MWE) at 6-month follow-up were significantly correlated with improvements in the magnitudes of conventional echocardiographic parameters $(\triangle \mathrm{LVESV} \%, \triangle \mathrm{LVEF}$, and $\triangle$ GLS, all $\mathrm{P}<0.001$, Table 3).

Also, the improved values of global MW indices $(\triangle \mathrm{GWI}$ and $\triangle \mathrm{GCW}$ ) at 6-month follow-up showed good correlations with the increased magnitudes of corresponding mid septal $M W$ indices $(\triangle \mathrm{mid}$ septal $M W I$ and $\triangle \mathrm{mid}$ septal $\mathrm{CW}, \mathrm{r}=0.772$ and $\mathrm{r}=0.789$, respectively, both $\mathrm{P}<0.001$, Figure 3).

\section{Analyses to identify baseline predictors of response to CRT}

Logistic regression analysis was performed to identify the independent predictors of response to CRT (Table 4). All variables significantly associated with CRT response in the univariate analysis were involved in the multivariate logistic regression analysis. In the multivariate analysis, baseline mid septal MWE [odds ratio (OR) 0.975, 95\% confidence interval (CI): 0.959-0.990, $\mathrm{P}=0.002]$ and mid lateral MWI (OR 1.003, 95\% CI: 1.002-1.004, $\mathrm{P}<0.001$ ) were identified as independent predictors of response to CRT.

ROC analysis identified the optimal cutoff value of mid septal MWE as $40 \%$ [sensitivity, 71\%; specificity, $71 \%$; area under the ROC curve $(\mathrm{AUC})=0.713, \mathrm{P}<0.001]$ and the optimal cutoff value of mid lateral MWI as $634 \mathrm{mmHg} \%$ 
Table 3 Correlations between changes in myocardial work indices and changes in conventional echocardiographic parameters at follow-up in comparison with baseline values

\begin{tabular}{|c|c|c|c|c|c|c|}
\hline Variable & \multicolumn{2}{|c|}{$\triangle$ LVESV \% } & \multicolumn{2}{|c|}{$\triangle \mathrm{LVEF}$} & \multicolumn{2}{|c|}{$\triangle G L S$} \\
\hline$\triangle \mathrm{GWI}$ & 0.619 & 0.000 & 0.631 & 0.000 & 0.843 & 0.000 \\
\hline$\triangle \mathrm{GCW}$ & 0.654 & 0.000 & 0.669 & 0.000 & 0.745 & 0.000 \\
\hline$\triangle G W E$ & 0.464 & 0.000 & 0.424 & 0.000 & 0.662 & 0.000 \\
\hline$\triangle$ Mid septal CW & 0.610 & 0.000 & 0.554 & 0.000 & 0.623 & 0.000 \\
\hline$\triangle$ Mid septal MWE & 0.547 & 0.000 & 0.446 & 0.000 & 0.472 & 0.000 \\
\hline
\end{tabular}

$\triangle$ indicates change; $\triangle$ LVESV\%, the percentage change in left ventricular end-systolic volume; GCW, global constructive work; GLS, global longitudinal strain; GWE, global work efficiency; GWI, global work index; LVEF, left ventricular ejection fraction; CW, constructive work; MWE, myocardial work efficiency; MWI, myocardial work index.
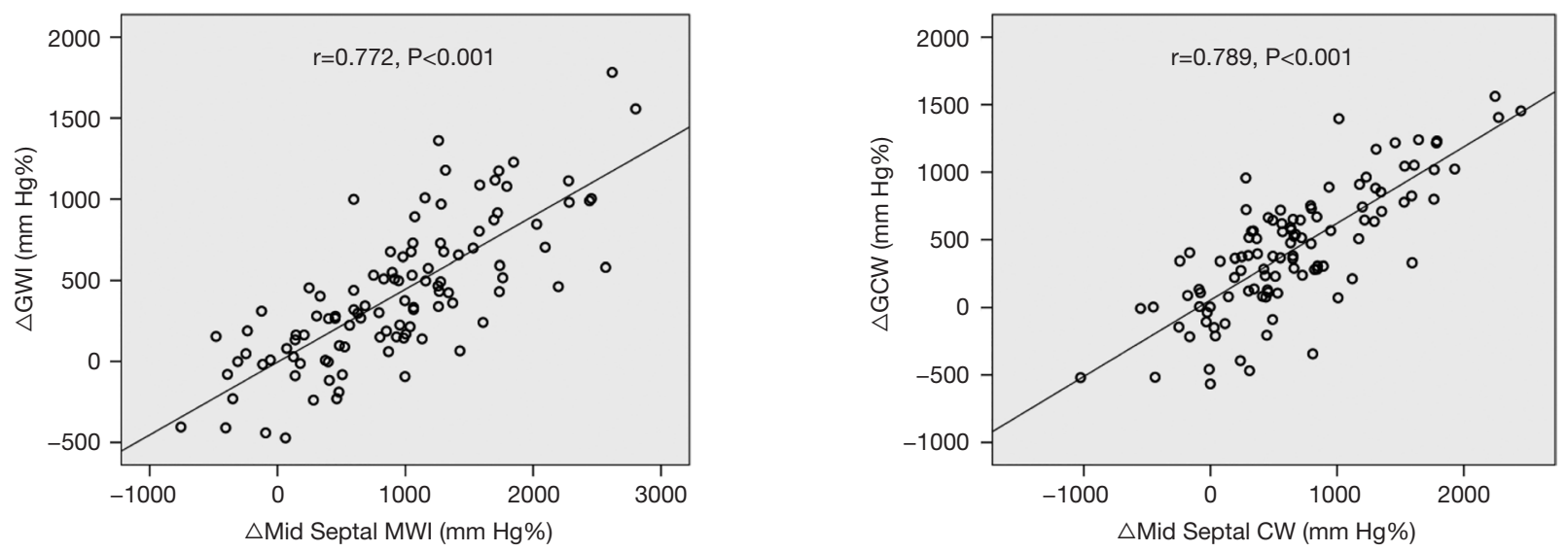

Figure 3 Correlations between changes in global MW indices and changes in mid septal MW indices. $\triangle$ indicates change; GCW, global constructive work; GWI, global work index; CW, constructive work; MW, myocardial work; MWI, myocardial work index.

(sensitivity, $85 \%$; specificity, $71 \%$; AUC $=0.807, \mathrm{P}<0.001$ ) for predicting response to CRT. The combination of the 2 predictors, mid septal MWE $\leq 42 \%$ and mid lateral MWI $\geq 740 \mathrm{mmHg} \%$, yielded sensitivity of $79 \%$, specificity of $82 \%$, and $\mathrm{AUC}=0.830(\mathrm{P}<0.001)($ Figure 4$)$.

\section{Discussion}

Non-invasive MW indices derived from LV PSLs, which were generated by combining LV LS measured by speckle tracking echocardiography with brachial artery blood pressure measured by sphygmomanometry, provide incremental clinical value for decision making in the selection of HF patients for CRT. In the present study, we evaluated the global and segmental MW indices in
CRT candidates and demonstrated that the abnormal but reversible segmental heterogeneous distribution of $\mathrm{MW}$ at baseline was significantly associated with favorable CRT response. The lower mid septal MWE and the higher mid lateral MWI at baseline were also identified as independent predictors of CRT response. Additionally, their combination could further improve the prediction accuracy of response to CRT, which indicates that the assessment of the potentially recoverable reserves in the septum and residual myocardial contractile performance in the lateral wall before CRT could be useful in the identification of responders.

The proportion of patients with coronary heart disease in our study population was not high. A degree of coronary artery stenosis can result in myocardial ischemia, and the 
Table 4 Analyses to identify the baseline predictors of positive response to CRT

\begin{tabular}{|c|c|c|c|c|c|c|}
\hline Variable & \multicolumn{3}{|c|}{ Univariate analysis } & \multicolumn{3}{|c|}{ Multivariate analysis } \\
\hline NYHA functional class IV & 0.241 & $0.082-0.71$ & 0.010 & & & 0.055 \\
\hline LBBB & 3.067 & $1.047-8.982$ & 0.041 & & & 0.593 \\
\hline DBP & 1.065 & $1.010-1.122$ & 0.020 & & & 0.447 \\
\hline LVESV & 0.990 & $0.984-0.996$ & 0.001 & & & 0.369 \\
\hline IVMD & 1.034 & $1.013-1.055$ & 0.002 & & & 0.061 \\
\hline GLS & 0.757 & $0.612-0.936$ & 0.010 & & & 0.497 \\
\hline Mid lateral LS & 0.827 & $0.746-0.917$ & 0.000 & & & 0.995 \\
\hline Mid lateral MWI & 1.003 & $1.001-1.004$ & 0.000 & 1.003 & $1.002-1.004$ & 0.000 \\
\hline Mid lateral CW & 1.002 & $1.001-1.003$ & 0.000 & & & 0.689 \\
\hline Mid septal MWI & 0.999 & $0.998-1.000$ & 0.006 & & & 0.386 \\
\hline Mid septal MWE & 0.969 & $0.950-0.988$ & 0.002 & 0.975 & $0.959-0.990$ & 0.002 \\
\hline Mid septal WW & 1.003 & $1.001-1.004$ & 0.000 & & & 0.312 \\
\hline
\end{tabular}

CRT, cardiac resynchronization therapy; Cl, confidence interval; CW, constructive work; DBP, diastolic blood pressure; GCW, global constructive work; GLS, global longitudinal strain; GWI, global work index; IVMD, interventricular mechanical delay; LBBB, left bundle branch block; LS, longitudinal strain; LVEDV, left ventricular end-diastolic volume; LVESV, left ventricular end-systolic volume; MW, myocardial work; MWE, myocardial work efficiency; MWI, myocardial work index; WW, wasted work; OR, odds ratio; NYHA, New York Heart Association.

etiology of heart failure may be attributed to the impact of myocardial ischemia over the long-term. Patients with severe myocardial ischemia have low myocardial viability and often fail to respond to CRT, especially those with a history of acute myocardial infarction, which develops into heart failure due to segmental wall motion abnormalities rather than dyssynchrony. For patients with significant coronary artery disease, percutaneous coronary intervention (PCI) is recommended over CRT.

Existing studies have also reported some predictors for CRT response. For example, Maass et al. (14) demonstrated that apical rocking and IVMD were significantly associated with the amount of reverse ventricular remodeling, and could predict clinical outcomes after CRT. Although visual assessment of apical rocking is a feasible and reproducible approach for identifying CRT responders, qualitative evaluation can be problematic for non-expert readers. IVMD was previously proposed to be useful for predicting CRT response (1); however, subsequent studies have produced conflicting results on its ability to predict CRT response. Most evidence suggests that IVMD is not as useful in predicting response to CRT (12). Previous studies have also shown that LBBB is associated with a favorable response to CRT. CRT responders in our study also exhibited a significantly higher prevalence of LBBB at baseline. However, in addition to the presence of $\mathrm{LBBB}$, residual LV myocardial viability, which represents the substrate for CRT response, is another important determinant of prognosis (15). Therefore, LBBB was not an independent predictor in our multivariate logistic regression analysis.

Invasive LV pressure-volume loops (PVLs) measured during coronary angiograms, as first demonstrated in experiments by Suga and Sagawa (16), have been used to quantify global LV function and segmental MW for over 40 years. However, the invasive LV pressure-volume/strain loops are not without risk to the patient. Developments toward a fully non-invasive assessment of MW were 


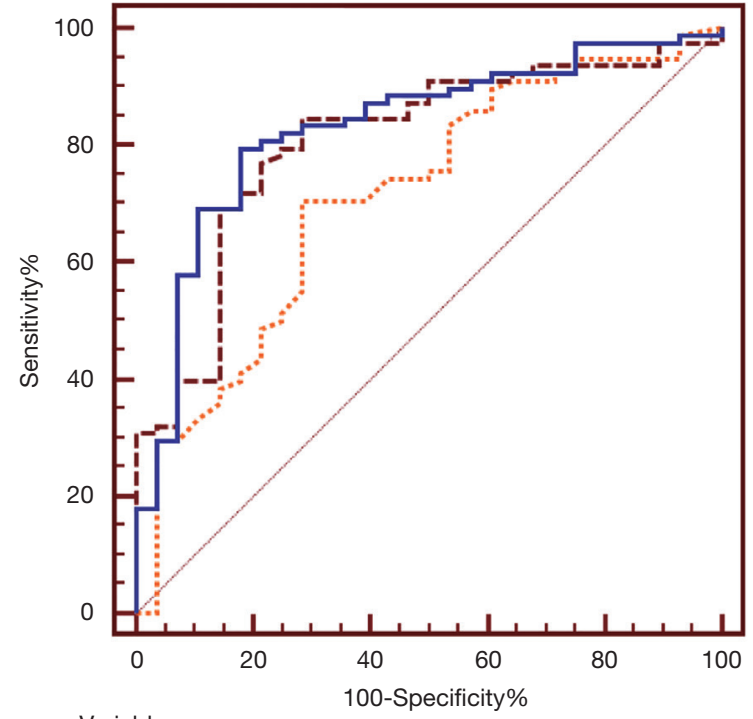

Variable

...... Mid Septal MWE

--- Mid Lateral MWI

_ Combine Mid Septal MWE with Mid Lateral MWI

AUC

0.713

0.807

0.830

Figure 4 ROC curves for predicting response to CRT. The area under the ROC curve (AUC) for combined mid septal MWE and mid lateral MWI was greater than that of mid septal MWE or mid lateral MWI. CRT, cardiac resynchronization therapy; ROC, receiver operating characteristic; MWE, myocardial work efficiency; MWI, myocardial work index.

therefore made in the hope of improving its clinical applicability. Russell et al. $(6,7)$ proposed a simple method to non-invasively estimate $L V$ pressure, which was based on the measurement of brachial artery cuff pressure and the timing of valvular events, then the LV strain data combined with this non-invasively estimated $L V$ pressure was used to construct LV pressure-strain loops (PSLs). The area within the non-invasive LV PSLs showed excellent correlation and good agreement with the invasive measurements $(6,7)$.

Recently, this non-invasive $M W$ analysis was introduced as a new vendor-specific module into the AFI software within the EchoPAC workstation (GE Vingmed Ultrasound), which is also the only currently available commercial system that can provide echocardiographic software to calculate MW. This new software has been applied under normal physiological conditions, and in some pathological LV remodeling. The Normal Reference Ranges for Echocardiography (NORRE) study (17), accredited by the European Association of Cardiovascular Imaging (EACVI), has provided useful normal reference ranges for novel non-invasive global $\mathrm{MW}$ indices. Furthermore, the NORRE sub-study (18) evaluated the correlations between global MW indices and traditional two-dimensional echocardiographic parameters, including LV size, and LV systolic and diastolic functional parameters. Considering that the study population was entirely composed of healthy subjects, leading to restricted LV size and function value ranges, these correlations were not all strong and were not observed for all MW indices. In our study with a HF population, the improved values for global and segmental MW indices at 6-month follow-up showed good correlations with the improved magnitudes of conventional echocardiographic parameters.

Moreover, the correlations in our study were stronger than those in the NORRE sub-study, as LV remodeling and dysfunction are usually tightly correlated, especially in advanced HF. Hedwig et al. (19) demonstrated the new GWI correlated with established prognostic parameters in HF patients. GWI $<500 \mathrm{mmHg} \%$ was a predictor of severely impaired ejection fraction, very low exercise capacity, and strongly elevated NT-pro-BNP, suggesting a poor prognosis. Also, non-invasive MW indices have shown more promising results in the diagnosis and prognostication of some cardiovascular diseases than strain alone (20-25).

The novel echocardiography-based technique for noninvasive MW calculation has been used in the field of CRT. Galli et al. (26) showed that HF patients with higher GCW exhibited a favorable response to CRT, which was consistent with our results that GCW was significantly higher in CRT responders than in non-responders at baseline evaluation. Van der Bijl et al. (27) demonstrated that lower GWE before CRT was independently associated with better longterm outcomes in HF patients with a Class I indication for CRT according to current guidelines. However, in our study, GWE was comparable between CRT responders and non-responders at baseline. Possible reasons for these contradictory results may include the various patient clinical characteristics, for example, their study population only consisted of patients with a Class I indication for CRT, but our study enrolled a wider range of patients.

The prognostic implications of segmental MW indices in HF patients undergoing CRT have not been further explored. Our study extended prior work to explore the value of segmental MW indices in predicting CRT response, and evaluated the changes in segmental MW indices after CRT. The focus was on the septal and lateral mid-ventricular levels because $\mathrm{LV}$ mapping data in typical $\mathrm{LV}$ dyssynchrony 
have shown that the mid septal region was electrically activated first and showed a U-shaped conduction pattern through the apical regions, with a prolonged activation time in the $\mathrm{LV}$ activation sequence, and was imposed between the LV septum and the lateral wall (28). Therefore, we believe that it enhances reproducibility since segmental heterogeneity is most evident between the septum and lateral wall, and mid-ventricular values could represent the complete myocardial wall and improve operability by simplifying the evaluation. In our study, CRT responders at baseline exhibited significantly lower mid septal MWI and mid septal MWE, and significantly higher mid lateral MWI and mid lateral CW than non-responders. The multivariate analysis identified baseline mid septal MWE and mid lateral MWI as independent predictors of CRT response. Baseline mid septal MWE, which presents potentially recoverable reserve, could play an important role in identifying possible responders to CRT. Baseline mid lateral MWI, which is an index of residual myocardial performance, is fundamental to CRT's success as myocardial substrates are related to the electrical response.

Dyssynchronous LV activation leads to unloading of the early activated regions, and a higher load in the late activated regions. As observed typically in patients with LBBB $(29,30)$, the early activated septum contracts before aortic valve opening under low LV pressure, stretching the LV lateral wall simultaneously. The late activated lateral wall contracts during rising $\mathrm{LV}$ pressures, thus increasing regional workload and causing a variable degree of systolic lengthening of the septum at the same time. Septal work during systolic shortening is absorbed by prestretching of the LV lateral wall, and work during systolic lengthening does not contribute to $\mathrm{LV}$ ejection, both of which represent a waste. Consequently, compensatory mechanisms increase contractility of the lateral wall. Inhomogeneity of contraction resulted in the widely varied contribution of the septum to $\mathrm{LV}$ work in CRT candidates. Vecera et al. (31) proposed an assessment of wasted MW in the septum as a novel method to identify patients who may be responders of CRT. Our results indicated that the less efficiently the septum operates at baseline, the greater the potentially recoverable reserve, which translates into a favorable outcome. CRT responders show the preservation of LV lateral wall function at baseline, making it possible to provide myocardial substrates for a favorable response to CRT. Increased scar burden or LV free wall fibrosis was found to be associated with poor response and worse outcomes after CRT $(32,33)$. Russell et al. (6) have shown that regional differences in MWI assessed by PSLs have a strong correlation with the myocardial glucose metabolism evaluated by $18 \mathrm{~F}-\mathrm{FDG}$ PET, which supports the hypothesis that the differences in MWI detected by PSLs before CRT might correspond to myocardial residual metabolic activity, and might therefore explain the role of baseline mid lateral MWI in predicting CRT response.

MW indices could also be used to examine the impact of treatment on LV function. Our present study corroborated the finding that CRT induces the dyssynchronous heart to a more balanced distribution of work, thereby improving global cardiac function significantly and demonstrated that the improvement in LV global function mainly resulted from the increase in septal work efficiency following CRT.

\section{Limitations}

First, for the non-invasive MW indices assessment, systemic arterial pressure was used as a substitute for $\mathrm{LV}$ pressure. This technique cannot be applied when systemic arterial pressure and LV pressure are discordant, for example, in patients with $\mathrm{LV}$ outflow obstruction or aortic stenosis, or any other cardiac pathologies that will induce a pressure gradient between the aorta and the LV. Second, atrial fibrillation, with significant beat-to-beat variability, inhibits the accurate and reliable assessment of MW indices in such patients. Third, this study is subject to the inherent limitations of a single-center, retrospective analysis. It would be ideal for retesting the cutoff values in a validation population. Larger scale multi-center studies are needed to evaluate the new parameters further to establish their clinical utility and prognostic implications in the field of CRT before they can be recommended as tools in clinical practice. Fourth, the non-invasive evaluation of MW was performed by estimating $\mathrm{LV}$ pressure, which is known to be imprecise. We are aware this method is somewhat imperfect; nevertheless, this parameter has the advantage of providing an automatic analysis of myocardial performance that is independent of $\mathrm{LV}$ afterload.

\section{Conclusions}

The novel echocardiography-based technique allows the non-invasive assessment of MW indices rapidly and effectively, and therefore has the potential to be widely used as a promising tool for selecting CRT candidates in clinical practice. Lower mid septal MWE and higher mid lateral MWI at baseline were identified as independent predictors 
of response to CRT, and their combination leads to more accurate and effective prediction than the individual parameters alone. Therefore, it is useful to improve patient selection for CRT to identify this abnormal but reversible segmental heterogeneity with the use of MW indices before CRT.

\section{Acknowledgments}

This manuscript has not been previously published or submitted for publication elsewhere except as a brief abstract in the proceedings of a scientific meeting or symposium. The abstract of our manuscript was submitted to the 2020 ASE 31st Annual Scientific Sessions for scientific exchange and was accepted as a poster presentation. All accepted original science presentations have been published in the June issue of Journal of the American Society of Echocardiography, 2020.

Funding: This work was funded by the National Nature Science Foundation of China (Grant No. 82071933).

\section{Footnote}

Conflicts of Interest: All authors have completed the ICMJE uniform disclosure form (available at http://dx.doi. org/10.21037/qims-20-754). The authors have no conflicts of interest to declare.

Ethical Statement: This study was approved by the medical ethics committee of Zhongshan Hospital, Fudan University, and informed consent was obtained from all patients.

Open Access Statement: This is an Open Access article distributed in accordance with the Creative Commons Attribution-NonCommercial-NoDerivs 4.0 International License (CC BY-NC-ND 4.0), which permits the noncommercial replication and distribution of the article with the strict proviso that no changes or edits are made and the original work is properly cited (including links to both the formal publication through the relevant DOI and the license). See: https://creativecommons.org/licenses/by-nc-nd/4.0/.

\section{References}

1. Cleland JG, Daubert JC, Erdmann E, Freemantle N, Gras D, Kappenberger L, Tavazzi L, Cardiac Resynchronization-Heart Failure Study I. The effect of cardiac resynchronization on morbidity and mortality in heart failure. N Engl J Med 2005;352:1539-49.

2. Bogale N, Priori S, Cleland JG, Brugada J, Linde C, Auricchio A, van Veldhuisen DJ, Limbourg T, Gitt A, Gras D, Stellbrink C, Gasparini M, Metra M, Derumeaux G, Gadler F, Buga L, Dickstein K, Scientific Committee NC, Investigators. The European CRT Survey: 1 year (9-15 months) follow-up results. Eur J Heart Fail 2012;14:61-73.

3. Mor-Avi V, Lang RM, Badano LP, Belohlavek M, Cardim NM, Derumeaux G, Galderisi M, Marwick T, Nagueh SF, Sengupta PP, Sicari R, Smiseth OA, Smulevitz B, Takeuchi M, Thomas JD, Vannan M, Voigt JU, Zamorano JL. Current and evolving echocardiographic techniques for the quantitative evaluation of cardiac mechanics: ASE/ EAE consensus statement on methodology and indications endorsed by the Japanese Society of Echocardiography. J Am Soc Echocardiogr 2011;24:277-313.

4. Kovács Z, Kormányos Á, Domsik P, Kalapos A, Lengyel C, Ambrus N, Ajtay Z, Piros G, Forster T, Nemes A. Left ventricular longitudinal strain is associated with mitral annular fractional area change in healthy subjectsResults from the three-dimensional speckle tracking echocardiographic MAGYAR-Healthy Study. Quant Imaging Med Surg 2019;9:304-11.

5. Park HE, Chang SA, Kim HK, Shin DH, Kim JH, Seo MK, Kim YJ, Cho GY, Sohn DW, Oh BH, Park YB. Impact of loading condition on the 2D speckle trackingderived left ventricular dyssynchrony index in nonischemic dilated cardiomyopathy. Circ Cardiovasc Imaging 2010;3:272-81.

6. Russell K, Eriksen M, Aaberge L, Wilhelmsen N, Skulstad H, Remme EW, Haugaa KH, Opdahl A, Fjeld JG, Gjesdal O, Edvardsen T, Smiseth OA. A novel clinical method for quantification of regional left ventricular pressure-strain loop area: a non-invasive index of myocardial work. Eur Heart J 2012;33:724-33.

7. Russell K, Eriksen M, Aaberge L, Wilhelmsen N, Skulstad H, Gjesdal O, Edvardsen T, Smiseth OA. Assessment of wasted myocardial work: a novel method to quantify energy loss due to uncoordinated left ventricular contractions. Am J Physiol Heart Circ Physiol 2013;305:H996-1003.

8. Ponikowski P, Voors AA, Anker SD, Bueno H, Cleland JGF, Coats AJS, Falk V, Gonzalez-Juanatey JR, Harjola VP, Jankowska EA, Jessup M, Linde C, Nihoyannopoulos P, Parissis JT, Pieske B, Riley JP, Rosano GMC, Ruilope LM, Ruschitzka F, Rutten FH, van der Meer P, Group ESCSD. 2016 ESC Guidelines for the diagnosis and treatment of acute and chronic heart failure: The Task Force for the diagnosis and treatment of acute and chronic 
heart failure of the European Society of Cardiology (ESC) Developed with the special contribution of the Heart Failure Association (HFA) of the ESC. Eur Heart J 2016;37:2129-200.

9. Brignole $M$, Auricchio A, Baron-Esquivias G, Bordachar P, Boriani G, Breithardt OA, Cleland J, Deharo JC, Delgado V, Elliott PM, Gorenek B, Israel CW, Leclercq C, Linde C, Mont L, Padeletti L, Sutton R, Vardas PE; ESC Committee for Practice Guidelines (CPG), Zamorano JL, Achenbach S, Baumgartner H, Bax JJ, Bueno H, Dean V, Deaton C, Erol C, Fagard R, Ferrari R, Hasdai D, Hoes AW, Kirchhof P, Knuuti J, Kolh P, Lancellotti P, Linhart A, Nihoyannopoulos P, Piepoli MF, Ponikowski P, Sirnes PA, Tamargo JL, Tendera M, Torbicki A, Wijns W, Windecker S; Document Reviewers, Kirchhof P, Blomstrom-Lundqvist C, Badano LP, Aliyev F, Bänsch D, Baumgartner H, Bsata W, Buser P, Charron P, Daubert JC, Dobreanu D, Faerestrand S, Hasdai D, Hoes AW, Le Heuzey JY, Mavrakis H, McDonagh T, Merino JL, Nawar MM, Nielsen JC, Pieske B, Poposka L, Ruschitzka F, Tendera M, Van Gelder IC, Wilson CM. 2013 ESC Guidelines on cardiac pacing and cardiac resynchronization therapy: the Task Force on cardiac pacing and resynchronization therapy of the European Society of Cardiology (ESC). Developed in collaboration with the European Heart Rhythm Association (EHRA). Eur Heart J 2013;34:2281-329.

10. Bleeker GB, Bax JJ, Fung JW, van der Wall EE, Zhang Q, Schalij MJ, Chan JY, Yu CM. Clinical versus echocardiographic parameters to assess response to cardiac resynchronization therapy. Am J Cardiol 2006;97:260-3.

11. Chung ES, Leon AR, Tavazzi L, Sun JP, Nihoyannopoulos P, Merlino J, Abraham WT, Ghio S, Leclercq C, Bax JJ, Yu CM, Gorcsan J, 3rd, St John Sutton M, De Sutter J, Murillo J. Results of the Predictors of Response to CRT (PROSPECT) trial. Circulation 2008;117:2608-16.

12. Gorcsan J, 3rd, Abraham T, Agler DA, Bax JJ, Derumeaux G, Grimm RA, Martin R, Steinberg JS, Sutton MS, Yu $\mathrm{CM}$, American Society of Echocardiography Dyssynchrony Writing G, American Society of Echocardiography Dyssynchrony Writing G, Heart Rhythm S.

Echocardiography for cardiac resynchronization therapy: recommendations for performance and reporting--a report from the American Society of Echocardiography Dyssynchrony Writing Group endorsed by the Heart Rhythm Society. J Am Soc Echocardiogr 2008;21:191-213.

13. Lang RM, Badano LP, Mor-Avi V, Afilalo J, Armstrong A, Ernande L, Flachskampf FA, Foster E, Goldstein SA,
Kuznetsova T. Recommendations for Cardiac Chamber Quantification by Echocardiography in Adults: An Update from the American Society of Echocardiography and the European Association of Cardiovascular Imaging. Eur Heart J Cardiovasc Imaging 2015;16:233-70.

14. Maass AH, Vernooy K, Wijers SC, van 't Sant J, Cramer MJ, Meine M, Allaart CP, De Lange FJ, Prinzen FW, Gerritse B, Erdtsieck E, Scheerder COS, Hill MRS, Scholten M, Kloosterman M, Ter Horst IAH, Voors AA, Vos MA, Rienstra M, Van Gelder IC. Refining success of cardiac resynchronization therapy using a simple score predicting the amount of reverse ventricular remodelling: results from the Markers and Response to CRT (MARC) study. Europace 2018;20:e1-e10.

15. Khidir MJH, Abou R, Yilmaz D, Ajmone Marsan N, Delgado V, Bax JJ. Prognostic value of global longitudinal strain in heart failure patients treated with cardiac resynchronization therapy. Heart Rhythm 2018;15:1533-9.

16. Suga H, Sagawa K. Instantaneous pressure-volume relationships and their ratio in the excised, supported canine left ventricle. Circ Res 1974;35:117-26.

17. Manganaro R, Marchetta S, Dulgheru R, Ilardi F, Sugimoto T, Robinet S, Cimino S, Go YY, Bernard A, Kacharava G, Athanassopoulos GD, Barone D, Baroni M, Cardim N, Hagendorff A, Hristova K, Lopez-Fernandez T, de la Morena G, Popescu BA, Penicka M, Ozyigit T, Rodrigo Carbonero JD, van de Veire N, Von Bardeleben RS, Vinereanu D, Zamorano JL, Rosca M, Calin A, Moonen M, Magne J, Cosyns B, Galli E, Donal E, Carerj S, Zito C, Santoro C, Galderisi M, Badano LP, Lang RM, Oury C, Lancellotti P. Echocardiographic reference ranges for normal non-invasive myocardial work indices: results from the EACVI NORRE study. Eur Heart J Cardiovasc Imaging 2019;20:582-90.

18. Manganaro R, Marchetta S, Dulgheru R, Sugimoto T, Tsugu T, Ilardi F, Cicenia M, Ancion A, Postolache A, Martinez C, Kacharava G, Athanassopoulos GD, Barone D, Baroni M, Cardim N, Hagendorff A, Hristova K, Lopez T, de la Morena G, Popescu BA, Penicka M, Ozyigit T, Rodrigo Carbonero JD, van de Veire N, Von Bardeleben RS, Vinereanu D, Zamorano JL, Rosca M, Calin A, Moonen M, Magne J, Cosyns B, Galli E, Donal E, Carerj S, Zito C, Santoro C, Galderisi M, Badano LP, Lang RM, Lancellotti P. Correlation between non-invasive myocardial work indices and main parameters of systolic and diastolic function: results from the EACVI NORRE study. Eur Heart J Cardiovasc Imaging 2020;21:533-41.

19. Hedwig F, Soltani S, Stein J, Schoenrath F, Potapov E, 
Knosalla C, Falk V, Knebel F, Knierim J. Global work index correlates with established prognostic parameters of heart failure. Echocardiography 2020;37:412-20.

20. Przewlocka-Kosmala M, Marwick TH, Mysiak A, Kosowski W, Kosmala W. Usefulness of myocardial work measurement in the assessment of left ventricular systolic reserve response to spironolactone in heart failure with preserved ejection fraction. Eur Heart J Cardiovasc Imaging 2019;20:1138-46.

21. Boe E, Russell K, Eek C, Eriksen M, Remme EW, Smiseth OA, Skulstad H. Non-invasive myocardial work index identifies acute coronary occlusion in patients with nonST-segment elevation-acute coronary syndrome. Eur Heart J Cardiovasc Imaging 2015;16:1247-55.

22. Edwards NFA, Scalia GM, Shiino K, Sabapathy S, Anderson B, Chamberlain R, Khandheria BK, Chan J. Global Myocardial Work Is Superior to Global Longitudinal Strain to Predict Significant Coronary Artery Disease in Patients With Normal Left Ventricular Function and Wall Motion. J Am Soc Echocardiogr 2019;32:947-57.

23. Galli E, Vitel E, Schnell F, Le Rolle V, Hubert A, Lederlin M, Donal E. Myocardial constructive work is impaired in hypertrophic cardiomyopathy and predicts left ventricular fibrosis. Echocardiography 2019;36:74-82.

24. Chan J, Edwards NFA, Khandheria BK, Shiino K, Sabapathy S, Anderson B, Chamberlain R, Scalia GM. A new approach to assess myocardial work by non-invasive left ventricular pressure-strain relations in hypertension and dilated cardiomyopathy. Eur Heart J Cardiovasc Imaging 2019;20:31-9.

25. Oberhoffer FS, Abdul-Khaliq H, Jung AM, Zemlin M, Rohrer TR, Abd El Rahman M. Assessment of left ventricular myocardial work in Turner syndrome patients: insights from the novel non-invasive pressure-strain loop analysis method. Quant Imaging Med Surg 2020;10:15-25.

26. Galli E, Leclercq C, Fournet M, Hubert A, Bernard A, Smiseth OA, Mabo P, Samset E, Hernandez A, Donal E. Value of Myocardial Work Estimation in the Prediction

Cite this article as: Zhu M, Wang Y, Cheng Y, Su Y, Chen H, Shu $\mathrm{X}$. The value of non-invasive myocardial work indices derived from left ventricular pressure-strain loops in predicting the response to cardiac resynchronization therapy. Quant Imaging Med Surg 2021;11(4):1406-1420. doi: 10.21037/qims-20754 of Response to Cardiac Resynchronization Therapy. J Am Soc Echocardiogr 2018;31:220-30.

27. van der Bijl P, Vo NM, Kostyukevich MV, Mertens B, Ajmone Marsan N, Delgado V, Bax JJ. Prognostic implications of global, left ventricular myocardial work efficiency before cardiac resynchronization therapy. Eur Heart J Cardiovasc Imaging 2019;20:1388-94.

28. Auricchio A, Fantoni C, Regoli F, Carbucicchio C, Goette A, Geller C, Kloss M, Klein H. Characterization of left ventricular activation in patients with heart failure and left bundle-branch block. Circulation 2004;109:1133-9.

29. Risum N, Strauss D, Sogaard P, Loring Z, Hansen TF, Bruun NE, Wagner G, Kisslo J. Left bundle-branch block: the relationship between electrocardiogram electrical activation and echocardiography mechanical contraction. Am Heart J 2013;166:340-8.

30. Risum N, Tayal B, Hansen TF, Bruun NE, Jensen MT, Lauridsen TK, Saba S, Kisslo J, Gorcsan J 3rd, Sogaard P. Identification of Typical Left Bundle Branch Block Contraction by Strain Echocardiography Is Additive to Electrocardiography in Prediction of Long-Term Outcome After Cardiac Resynchronization Therapy. J Am Coll Cardiol 2015;66:631-41.

31. Vecera J, Penicka M, Eriksen M, Russell K, Bartunek J, Vanderheyden M, Smiseth OA. Wasted septal work in left ventricular dyssynchrony: a novel principle to predict response to cardiac resynchronization therapy. Eur Heart J Cardiovasc Imaging 2016;17:624-32.

32. Birnie D, DeKemp RA, Ruddy TD, Tang AS, Guo A, Williams K, Wassenar R, Lalonde M, Beanlands RS. Effect of lateral wall scar on reverse remodeling with cardiac resynchronization therapy. Heart Rhythm 2009;6:1721-6.

33. Riedlbauchová L, Brunken R, Jaber WA, Popova L, Patel D, Lanska V, Civello K, Cummings J, Burkhardt JD, Saliba W, Martin D, Schweikert R, Wilkoff BL, Grimm $\mathrm{R}$, Natale A. The impact of myocardial viability on the clinical outcome of cardiac resynchronization therapy. J Cardiovasc Electrophysiol 2009;20:50-7. 